\title{
La Desigualdad Social: Desarkollo y Desafíos del Trabajo Social DESDE LA RECONCEPTUALIZACIÓN EN AMÉRICA LATINA
}

\author{
Por: Norberto Alayón \\ María Lorena Molina
}

\section{RESUMEN}

Este artículo (síntesis de la Ponencia presentada al $33^{\circ}$ Congreso Mundial de Escuelas de Trabajo Social-Chile 2006) aspira responder a la pregunta: ¿Cuáles son las principales rupturas que se gestan en el Movimiento de Reconceptualización del Trabajo Social y cuáles los principales ejes de debates que influencian la formación profesional?

Paraconstruirnuestrasrespuestasplanteamosloqueimplicacolocarseen lacomprensión de las "problemáticas sociales" o bien trascender hacia las "manifestaciones de la cuestión social". Apuntamos hacia la relación de la política social con los derechos humanos y la ciudadanía, para superar la comprensión de meros instrumentos de control, mecanismos redistributivos y de estímulo al consumo, para identificarlas también como terreno estratégico de arena de conquista, ampliación y exigibilidad de derechos sociales. Todo esto lo vinculamos con el debate teórico-metodológico-ético político actual.

El texto concluye con algunas sugerencias acerca de posibles temas, preguntas o áreas de reflexión, según nuestro juicio medulares para continuar el debate sobre el Trabajo Social en la Latinoamérica contemporánea.

PALABRAS CLAVES: Formación profesional, Trabajo Social, desigualdad social, Reconceptualización, rupturas.

\section{ABSTRACT}

This article (Its a synthesis of the report, present to the $33^{\circ}$ World congreso of social work colleges-Chile 2006) desire to answer this question: iwhich are the main point of breaking that generate the Movement of Reconception of the Social Work profession an also wich are the main core of discussion that influence the education in the social work profession?. 
For create the answer, we have to understand "The social problems" or to be transcendent with the social manifestations: we want to relate the social politics with the human rights and citizenship, for generate knowledge to the control instruments, and the institutions in charge of the economic trends and the consumption. The social workers have to learn how to deal with this kind of knowledge and also demand to fulfil the social right. All this is related with the discuss of the theory-metodology-ethical and politics of the actuality.

The text conclude with the suggestion of possible topics or subjects to thinks about to continue the discuss of social work today in Latinoamerica.

KEYWORDS: Professional education, social work, social innegualities, Reconception of the Social Work profession.

\section{INTRODUCCIÓN}

$\longrightarrow$ debate generado por los y las participantes en el XVIII Seminario Latinoamericano de Escuelas de Trabajo Social (llevado a cabo en San José, Costa Rica en julio de 2004), referido a: La cuestión social, las políticas sociales y la formación profesional en el contexto de las nuevas relaciones de poder y la diversidad latinoamericana, colocó el tema de la Reconceptualización y sus repercusiones en la profesión como eje de debate. Sin embargo esto apenas quedó esbozado en este foro académico. A partir de allí el profesor Alayón se da a la tarea de indagar sobre cuatro preguntas claves a saber: a) qué fue y qué significó el Movimiento de Reconceptualización; b) qué características tuvo en cada país; c) cuánto y como influenció la formación y la práctica profesional en sus inicios y décadas siguientes; y d) qué vigencia tiene dicho movimiento, en la actualidad para los y las profesionales en trabajo social. Los resultados de su indagación en los países de América Latina dio lugar a la organización del libro: Trabajo Social Latinoamericano - A 40 años de la Reconceptualización, publicado por Espacio Editorial de Buenos Aires.

Las tendencias de los debates contemporáneos sobre el Trabajo Social en América Latina y la lectura del texto a juicio de Lorena Molina, posibilita una reflexión más amplia y vinculada al tema del $33^{\circ}$ Congreso Mundial de Escuelas de Trabajo Social, realizado en Santiago de Chile en agosto de 2006. Por ello, puede afirmarse que los textos de las y los autores por países que resultaron de la investigación de Alayón pueden ser analizados y derivar de ellos temas atinentes al Congreso Mundial y arribar a conclusiones que den origen a nuevas preguntas de investigación de ámbito regional o sub-regional o bien, a una agenda de debate latinoamericano que dé sostenibilidad a la rearticulación de las unidades 
académicas. En este sentido, hemos estimado pertinente compartir estas reflexiones y conclusiones que pueden dar lugar a una agenda de discusiones futuras entre unidades de enseñanza y gremios.

El Trabajo Social latinoamericano ha tenido inflexiones, rupturas desde la Reconceptualización, en particular a partir de una lectura diferente, crítica de la desigualdad social. Recuperar nuestra historia latinoamericana como contexto particular, inscrito en la historia general de la humanidad, para rescatar y resignificar la trascendencia del Movimiento de Reconceptualización es una de nuestras motivaciones fundamentales. Compartimos que este movimiento no es una etapa que pasó, sino un movimiento en desarrollo y que desde sus contradicciones hoy se manifiesta anhelando superar las insuficiencias y omisiones en medio de las rupturas y de las continuidades.

Pensamos que plantear la pertinencia contemporánea de una perspectiva crítica para interpretar los procesos socio-históricos desde donde el Trabajo Social es delimitado y a su vez delimita sus intervenciones profesionales, necesariamente nos remite a este Movimiento de ruptura. Posicionarnos hoy en el desafío de hacer rigurosas investigaciones de las manifestaciones de la cuestión social y de los sujetos que las viven como violaciones a sus derechos humanos -comprendidos en el entretejido social- para construir las intervenciones del singular-particularhumano en su diversidad, es un desafío clave.

Hoy constituye un reto fundamental orientar la formación y el quehacer profesional en consonancia con una férrea lucha por la promoción, ampliación, defensa y exigibilidad de los derechos humanos, ya sea desde la acción profesional directa con las poblaciones meta de los servicios sociales, con el trabajo socioeducativo promocional con las organizaciones de la sociedad civil constructor de ciudadanías y desde las competencias en el campo de la gestión y formulación de las políticas sociales.

\section{El Trabajo Social en el contexto de las Relaciones Sociales}

Presentaremos algunos puntos de vista que tiendan a caracterizar el quehacer del Trabajo Social en el contexto de las relaciones sociales que predominan en nuestras sociedades, identificando básicamente los aspectos centrales de la actual coyuntura en la que debe desenvolverse la actividad profesional. A partir de esto, se puede reconocer y comprender el perfilamiento de las orientaciones y objetivos de la profesión y las continuidades y rupturas que se registraron en el proceso histórico de su constitución. 
Fue durante el pasado siglo XX la época en que se instituyó y cristalizó la profesión en América Latina en el contexto del desarrollo del capitalismo monopólico y la constitución del Estado Benefactor. Por lo tanto, la formación de profesionales está marcada por esta condición histórica, conformando, así, un colectivo profesional específico dedicado a las complejas tareas de la acción social.

En escasos 70 u 80 años esta joven profesión evidenció cambios espectaculares en la lectura de la conformación de las dinámicas sociales latinoamericanas, como en la orientación y formación de sus cuadros y en las prácticas de acción concretas, marcadas por el auge del desarrollo industrial y la decadencia de los modelos agroexportadores de las economías latinoamericanas.

Durante el proceso de industrialización en los países del Cono Sur y sustitución de importaciones en Centroamérica son evidentes las consecuencias sociales que configuraron el escenario de las manifestaciones de la cuestión social, reseñadas brevemente en: migraciones del campo a la ciudad, los crecientes cinturones de miseria, los conflictos agrarios por la tenencia de la tierra, los efectos de la proletarización en el agro y en los centros urbanos, y toda la complejidad social que ello conlleva. Como respuestas a esta efervescente lucha de las y los trabajadores, las respuestas estatales van configurando una fragmentada política social en algunos países y en otros una restringida intervención estatal ante las crecientes manifestaciones de la cuestión social.

En este escenario en los foros promovidos por ALAETS (Asociación Latinoamericana de Escuelas de Trabajo Social) durante dos décadas, encontramos la ruptura con la tesis conservadora del Trabajo Social de raíces positivistas, pragmáticas y de marcada influencia norteamericana. Todo ello expresado en el Movimiento de Reconceptualización del Trabajo Social latinoamericano.

\section{Contribuciones y Limitaciones del Conocimiento de Reconceptualización}

En los años 70 parecían inminentes los cambios revolucionarios, y la posibilidad de construcción de una nueva sociedad -exenta de dominaciones externas e internas y pletóricas de legítimos ideales de justicia social- sobrevolaba los cielos de la América expoliada.

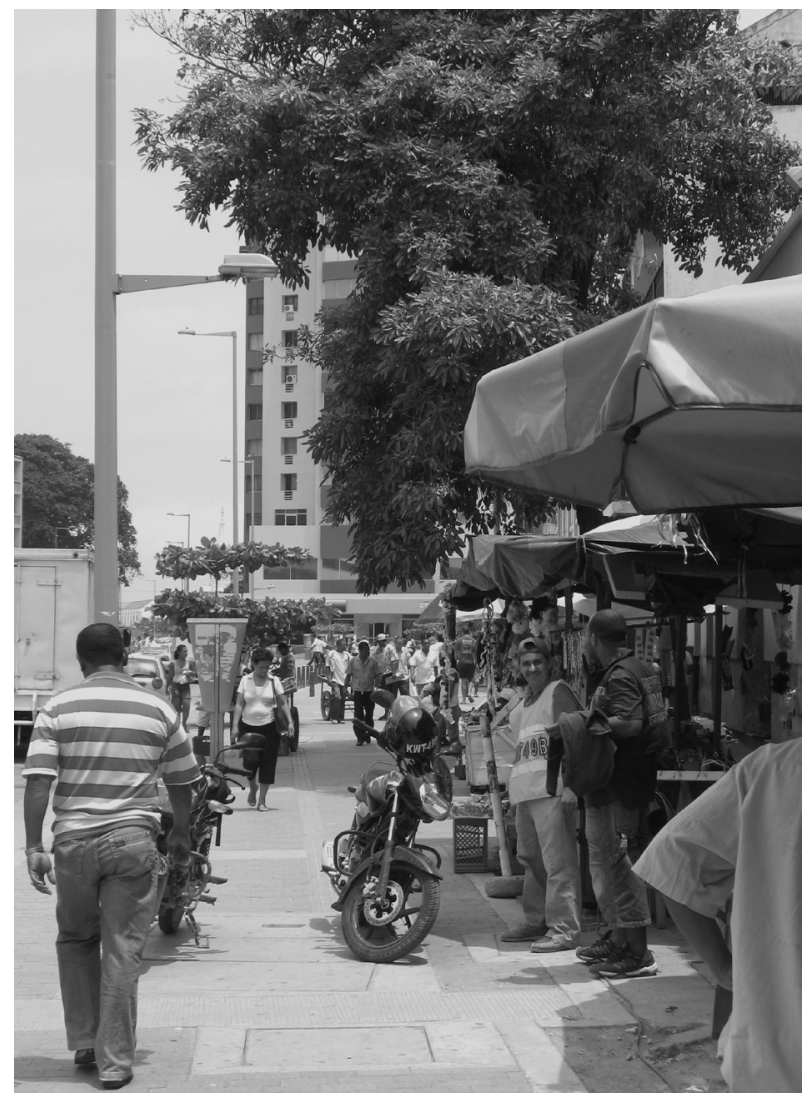


Y ello, como no podía ser de otra manera, impregnó al conjunto de las ciencias sociales y también, en particular, a nuestra profesión. En esa época se hablaba, se escribía y se discutía fuertemente acerca de una sociología de la liberación.

Sin embargo, no se trata de mirar a la Reconceptualización sólo con indulgencia o mero romanticismo. Se fueron gestando entonces -al calor de la efervescencia, del infortunio de la época y de las utopías liberadoras- aportaciones muy significativas para la profesión, aunque, a la distancia, también se identificaron serias limitaciones.

El compromiso y la responsabilidad social de los intelectuales en pos del mejoramiento de las sociedades, atraviesa todas las épocas. El filósofo Alain Badiou nos recordaba en 2004 que el intelectual tiene que ser un actor, un militante de la verdad, un combatiente. No somos testigos del mundo; tenemos que incorporarnos al devenir, con frecuencia paradójico y violento, de las verdades, ya sea que esas verdades sean políticas, artísticas, amorosas o científicas.

Los anhelados cambios revolucionarios de los 70 no se produjeron o bien fueron abortados abiertamente por violentos procesos locales, algunos con ayuda externa, que respondían a los intereses de siempre, contrarios a la autonomía y al bienestar general de nuestros pueblos.

En ese fragor, las y los trabajadores sociales latinoamericanos comenzamos a develar, aún incompletamente y con desaciertos, cuestiones centrales para nuestro quehacer profesional a partir de una creciente familiarización con la teoría. El colega uruguayo Herman Kruse críticamente observaba en 1971 que Tradicionalmente el servicio social cultivó un olímpico desprecio por la teoría. Todo el énfasis se centraba en la práctica.

El análisis de los procesos estructurales que influyen decisivamente-aunque no en forma absoluta en todos los casos y situaciones- en la cotidianeidad de los sujetos con los cuales interactuamos los trabajadores sociales, constituyó y constituye un significativo aporte para la comprensión del quehacer profesional.

Emergen las pugnas internas en las escuelas que asumen el debate por un pensamiento hegemónico en la comprensión del Trabajo Social que se posiciona en un repliegue de parte de algunos y en otros por avanzar en la discusión.

Pero a su vez, germina la discusión acerca del eje de las políticas sociales en la década del 70 estimulados desde el CELATS (Centro Latinoamericano de Trabajo Social) y tiempo después sobre los derechos humanos y la no discriminación, 
en la formación académica. A mitad de la década del 70 se posiciona el debate sobre las políticas sociales y en 1986 ALAETS (Asociación Latinoamericana de Escuelas de Trabajo Social) convoca al XIII Seminario Latinoamericano de Escuelas de Trabajo Social con la temática de la democracia, los derechos humanos, la participación y el Trabajo Social.

Sin duda alguna, la Reconceptualización marca un hito fundamental en la forma de entender hoy la historia y razón de ser de esta profesión en asuntos referidos al cuestionamiento a la tradición evolucionista, explicativa del origen de la profesión; al soporte empirista naturalizador de los problemas sociales; a la intervención atomizada o pulverizada desde la acción estatal. Estas problematizaciones marcan según nuestro criterio, una de las principales contribuciones para fraguar la génesis de un pensamiento crítico del Trabajo Social.

La significativa importancia que tuvo el proceso de Reconceptualización en el desarrollo del Trabajo Social latinoamericano, torna imprescindible y absolutamente actual la necesidad de recuperar el análisis del mismo, tanto en su génesis como en la influencia posterior que se verificó en la formación y en la práctica profesional de las y los trabajadores sociales.

Volver a analizar y discutir el significado y las características del Movimiento de Reconceptualización nos parece importante a la luz de la realidad actual, en la cual la consumación de verdaderas y profundas revoluciones conservadoras que se registraron en las últimas décadas en América Latina, nos confronta a los trabajadores sociales con la brutal restricción o pérdida lisa y llana de los derechos sociales históricamente conquistados, lo cual modificó el escenario de la práctica profesional.

Entre aquella época y condiciones y el presente de la profesión, se registra también un importante crecimiento y reconocimiento del Trabajo Social que, entendemos, en parte es tributario de las redefiniciones propuestas, primero, por el desarrollismo, y reelaboradas luego por el movimiento reconceptualizador, en el contexto de las expectativas políticas antes señaladas.

¿En qué medida puede reconocerse una deuda de la profesión con el Movimiento de Reconceptualización en los avances logrados y dónde radican, a su vez, las limitaciones de las propuestas de este movimiento, como para no haber dado lugar a un proyecto diferenciado pero viable para la profesión? Entre esas limitaciones, ¿cuáles corresponden a su debilidad teórica y cuánto de la inviabilidad de su desarrollo se debe, a su vez, a las condiciones políticas de nuestros países, que reprimieron todo pensamiento crítico, frustrando, así, aquella posibilidad? 
En el intento de responder a estas preguntas y de reconstruir el presente como momento de un proceso inacabado, nos propusimos este balance del acontecimiento más significativo de la historia del Trabajo Social, ya que -aún con todos sus límites- conmovió fuertemente a la profesión y los supuestos en los que ésta se sostenía.

De ahí la necesidad de seguir analizando y reflexionando sobre la Reconceptualización no por el simple regocijo de una especie de historicismo abstracto, sino para contribuir a dilucidar las posibilidades y los límites del Trabajo Social ante la gravedad de la situación social actual. Creemos que es este un modo de contribuir también a iluminar el presente de la profesión.

Porque la Reconceptualización, entendida como reflexión y acción crítica, no ha desaparecido; de la misma manera que -a pesar de ser esta una época de marcado retroceso- tampoco han desaparecido los ideales por la vigencia de una sociedad y un mundo más libres y más justos.

Porque la certeza de que las utopías de dignidad no han fenecido, mantiene la imperecedera llama que da fuerza a las luchas actuales y futuras en pos de los cambios deseados.

Porque si estas sociedades deshumanizadas son, obviamente, una construcción social, producto de los hombres y las mujeres que las componen; también las mujeres y los hombres tenemos la absoluta posibilidad (¿y la obligación?), aún en condiciones marcadamente subordinadas pero no definitivamente ineluctables, de construir otro tipo de sociedades, basadas en relaciones de solidaridad, fraternidad y equidad.

Y para ello entonces, como siempre, las y los trabajadores sociales seguimos contando con nuestra palabra y con nuestra acción para contribuir, humilde pero vitalmente, a ese digno proceso de bregar por el mejoramiento de la sociedad.

En el plano de la formación académica, asumir el legado de la Reconceptualización es un desafío crucial. Es fundamental formar futuros profesionales de pensamiento, acción crítica, propositiva y con compromiso ético político surgido del estudio de la cotidianidad como expresión histórica en cada contexto de país y con capacidad estratégica para:

- Investigar las manifestaciones y la configuración de la cuestión social con las transformaciones en el mundo del trabajo (producción) y de las familias (reproducción social) en el marco de las relaciones sociales marcadas por la 
sociedad patriarcal y la lógica capitalista como punto medular para construir la intervención profesional desde el Trabajo Social.

- Comprender a los seres humanos en su universalidad y particularidad, así como la diversidad que hay en ellos y las formas de discriminación que viven por criterios: socio-clasistas, de género, étnicos, de edad, opción sexual, religión, etc.

- Involucrarse y comprender las respuestas desde las organizaciones y movimientos sociales en la lucha por la vida.

- Asumir con criticidad las respuestas fragmentadas desde la política social en la lógica neoliberal, en el manejo de la cotidianidad de las familias incluidas y excluidas en los procesos de trabajo con y sin protección social estatal.

- Posicionarse en los espacios de defensa de los derechos como profesional orgánico a favor de los intereses de la mayoría, pero entendiendo el juego de poder institucional para argumentar cambios en la formulación, gestión y ejecución de las políticas sociales.

- Ser parte de la formulación de proyectos de ley o bien articularse a las organizaciones de la sociedad civil para incidir en la política o en los cambios a la ley.

En síntesis: asumir un perfil profesional comprometido con los valores éticohumanistas: igualdad, justicia y libertad, como condición para la construcción de sujetos individuales y colectivos cuestionadores de la historia construida, que los subordina a formas de existencia negadoras de la dignidad humana, para constituirse en ciudadanos creadores de una historia social signada por un mundo incluyente. Esto implica la construcción de una nueva ciudadanía y la profundización de la democracia.

En los años de la génesis y del desarrollo de las primeras décadas de la profesión, conocida como la perspectiva clásica del Trabajo Social, la comprensión de los objetos de intervención se correspondió con el concepto de problemáticas sociales entendidas como disfuncionalidades de un sistema social, en las cuales los sujetos cargan con una responsabilidad mayor, esto es el carácter privado del problema. Tal problema pese a ser social es individualizado en los procesos de intervención. Esta categoría y los presupuestos epistemológicos que subyacen en ella, constituyeron uno de los meollos de la crítica en el Movimiento de Reconceptualización, en tanto que refiere a una concepción de realidad social que naturaliza los hechos 
y procesos sociales o sea los acepta y no son objeto de transformación; sólo lo son de modificación para "re-encajar" en las condiciones sociales existentes que le dan origen. En forma congruente con esta noción, la concepción de individuo y sus acciones está descontextualizada de sus relaciones sociales.

La perspectiva crítica del Trabajo Social gestada en este movimiento, si bien no coloca en el inicio la categoría cuestión social, sí ubica los objetos de intervención profesional en la comprensión histórico materialista de la sociedad. Años más adelante, se plantea el tema de la cuestión social y sus manifestaciones como la plataforma fundamental para comprender la profesión en la historia. Así, como apunta Iamamoto (2003), la cuestión social no es pensada sólo como desigualdad, ni como las situaciones problemas reducidas a dificultades del individuo. La cuestión social conforma la materia prima del trabajo profesional. Es comprendida desde la génesis de las desigualdades sociales en un contexto donde el capital no rima con las equidades. Descifrarla también implica reconocer las formas de lucha, resistencia material y simbólica.

Como señala Paulo Netto (1997), el giro al carácter público que adquiere la atención de las manifestaciones de la cuestión social es determinado por: las exigencias económico-sociales; el protagonismo de los movimientos político sociales de los trabajadores y por el dinamismo político cultural asociado a las

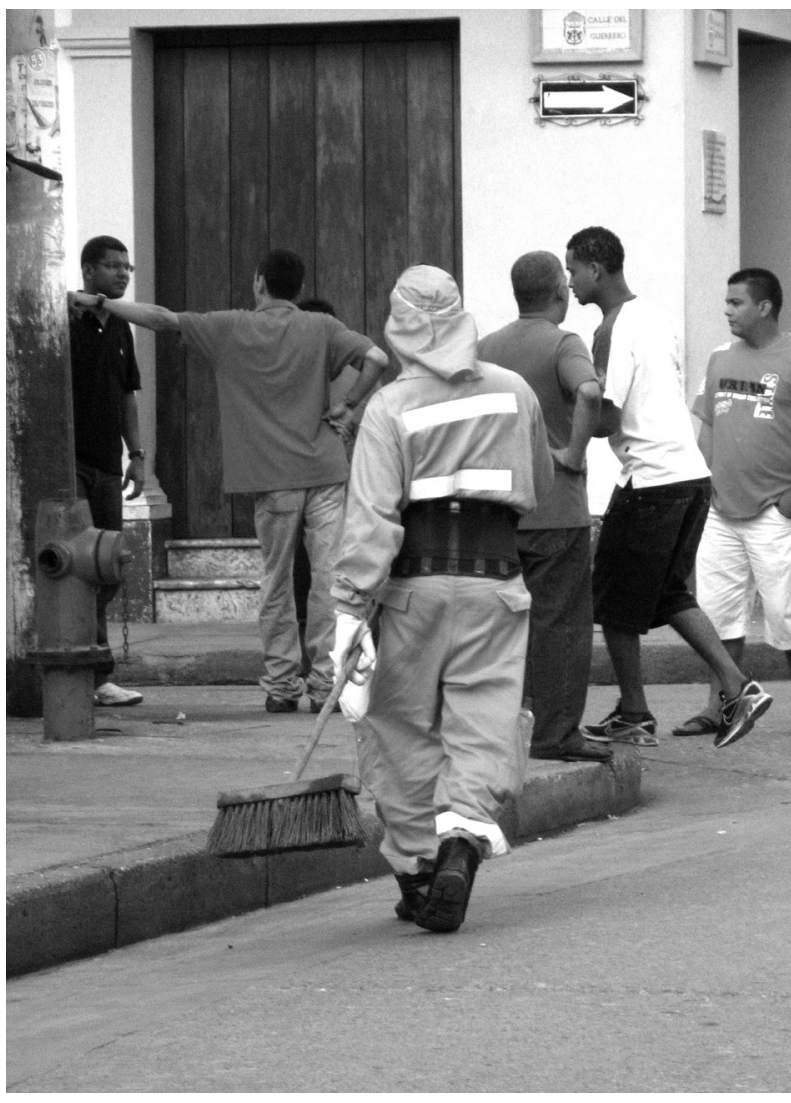
fundamental refiere a la comprensión de la realidad social como resultado histórico y a la noción de sujeto como producto y artífice de la historia individualsocial.

\section{La Profesión Social, Dimensión Ética del Trabajo Social}

El encaje en las condiciones pre-existentes buscado en la acción profesional es superado por la comprensión de la acción transformadora desde el trabajo profesional, el cual hoy es entendido en el marco de la defensa, la protección, la promoción y la exigibilidad de los satisfactores que hacen posible la construcción de equidades sociales, según corresponde al enfoque de los derechos humanos en su integralidad e indivisibilidad. Ello refiere a colocar en la brújula del trabajo profesional el respeto y la integralidad de los derechos civiles y políticos que resumen el derecho a 
la vida, la seguridad, la no violencia y a la participación ciudadana; los derechos económicos y sociales que adjetivan el derecho a la vida con dignidad, en tanto refieren al derecho al trabajo con la seguridad social, la salud, la vivienda, la educación y los derechos de tercera generación referidos a la autodeterminación de los pueblos, la cultura, la paz y a un ambiente sano.

Entendemos que la superación de la desigualdad social no será resultado de la acción de las profesiones, sino resultado de las luchas y movimientos sociales y políticos que transforman la historia. Por lo tanto no es la transformación social en este sentido, como hoy entendemos, la meta del trabajo profesional, pero sí el norte de la dimensión ética, o sea aportar a construir un mundo inclusivo.

Si asumimos que en la historia está la fuente de nuestros problemas y también en ella la búsqueda de las soluciones y entendemos que, como profesionales nos posicionamos en una ética expresada en la defensa, promoción, protección y exigibilidad de los derechos humanos (aunque la ley no necesariamente sea expresión de equidad en su realización), los parámetros de la intervención se reconfiguran. No es lo mismo, diagnosticar desde un enfoque de necesidades/ carencias de satisfactores -porque cabe interrogarse: ¿Cuáles son los parámetros de referencia: la conciencia de las carencias del sujeto o los parámetros del profesional para decidirle al otro lo que necesita?- o posicionarse en el enfoque de derechos humanos para diagnosticar lo que se violenta, lo que no se accede $y$ ante lo cual corresponde generar mecanismos de exigibilidad (individuales, familiares, grupales, organizacionales, barriales, etc.). Todo esto implica forjar conciencia de ciudadanía ampliada, consecuente con la radicalización de la democracia en el significado que le atribuye Agnes Heller.

Los derechos humanos se configuran en un discurso estratégico para la acción con los desfavorecidos del sistema, a partir de las exigencias de la cotidianidad de los sujetos sociales, en tanto son producto histórico. Por ello, esa cotidianidad debe ser comprendida como expresión de determinantes económicos, sociales y culturales construidos en cada realidad y en su reproducción individual, familiar, local.

No proponemos, en modo alguno, renunciar a la lucha por la generación de cambios más estructurales. Pero, a la vez, es absolutamente necesario (y eso está al alcance de la acción profesional) no desatender las acciones que producen cambios y mejoramientos (que a veces parecen menos relevantes) en la capacidad de las instituciones para hacer efectivos los derechos.

Todos estos avances y rupturas fueron posibles a partir de la semilla de la Palubrano. 9 Agosto/2008 
autocrítica, nacida allí, en este Movimiento de Reconceptualización. Este movimiento tuvo un desarrollo diverso en su primera década y nos llevó a planteamientos simplistas y negadores de la historia misma, los cuales se resumen en el mesianismo -ilustrado con el "rol de agente de cambio"- y el fatalismo, inmovilizador y negador de la capacidad creativa derivado de la comprensión instrumentalista, mecánica del papel del Estado y de la inserción que en él hace el trabajo profesional. Sin embargo, lo cierto es que es la cuna del pensamiento crítico del Trabajo Social. Las desviaciones en el estudio de un marxismo sin Marx (Consuelo Quiroga), nos condujeron a la bizantina discusión referida a que el materialismo histórico nos explica el macro entorno y las causas estructurales de las problemáticas desde donde se inician las intervenciones profesionales, pero resulta insuficiente para la intervención en el mundo microsocial y cotidiano. De allí en adelante, vinieron las marchas y las contramarchas y la restauración de los métodos clásicos y la vuelta a psicologización de las manifestaciones de la desigualdad social. Creemos que al estudiar un marxismo sin Marx, nos quedamos atrapadas y atrapados.

Hoy pensamos que si pudiésemos retornar al estudio de la dialéctica de la naturaleza humana, la ontología del ser social desde la tradición marxista, la teoría del valor del trabajo, así como desarrollar investigación sostenida sobre las manifestaciones de la cuestión social desde donde se configuran los objetos particulares de la intervención profesional, sobre cómo hemos realizado las intervenciones sociales, podríamos avanzar, pues conviene trascender los muros que nos aprisionan cuando sólo miramos en sí misma la profesión, pero sobre todo por la responsabilidad ético político que implica soñar un mundo con equidad.

Concluimos señalando que entre las contribuciones sobresalientes en el debate académico, ALAETS ha estimulado los temas sobre la formación profesional intrínsecamente articulada a las particularidades de nuestra América Latina.

A nuestro juicio, algunos de los ejes de análisis que son pilares en la constitución de la currícula y que marcan una direccionalidad o un sentido ético-político de la formación y el trabajo profesional y que requieren ser profundizados podrían ser:

- De la comprensión de las problemáticas sociales a las manifestaciones de la cuestión social y su implicación en la intervención profesional en términos de direccionalidad ético-político, teórico metodológico y técnico operativo.

- Del asistencialismo hacia la exigibilidad de los derechos humanos y la comprensión del papel contradictorio de las políticas sociales y sus implicaciones 
en la formulación, gerencia y ejecución de programas sociales.

- De la perspectiva evolucionista de la asistencia, hacia la perspectiva crítica de la génesis de la profesión en la historia social y política de los países de Latinoamérica.

- De la dimensión técnico-operativa en la intervención, hacia una perspectiva crítica e integral de las dimensiones teórico-metodológicas y ético político expresada en los programas de los cursos o materias.

- De la comprensión de métodos auxiliares de la investigación y la administración, a la perspectiva de elementos constitutivos de la dimensión teórico metodológica y técnico operativa y su implicación en los cursos o materias para la formación profesional.

- De las prácticas académicas reproductoras a las prácticas académicas críticopropositivas comprendidas en el contexto de los factores y mediaciones constitutivas del trabajo profesional.

- De la comprensión de la categoría práctica profesional centrada en la dimensión técnico operativa hacia la comprensión de la categoría trabajo profesional.

- De una comprensión de la moral y ética humanista abstracta a una dimensión ética y política comprometida con la superación de las inequidades y sus implicaciones, no sólo en la comprensión sino también en el desarrollo de actitudes pertinentes con el respeto y la no discriminación de los seres humanos

\section{BIBLIOGRAFIA}

ALAYÓN, Norberto. (1980). El asistencialismo en la Política Social y en el Trabajo Social. En: Revista Acción Crítica No.7. CELATS-ALAETS. Lima.

. (1980). Hacia la historia del Trabajo Social en Argentina CELATS.

Lima.

(1988). Perspectivas del Trabajo Social. Hvmanitas. Buenos

Aires.2da. edición.

Palubrano. 9 Agosto/2008 
- (1989). Asistencia y asistencialismo. ¿Pobres controlados o erradicación de la pobreza? Hvmanitas. Buenos Aires. (3ra. edición aumentada. Buenos Aires. Lumen. 2000).

. (2004). Dimensión política del Trabajo Social. En: Revista Escenarios No. 8. Escuela Superior de Trabajo Social de La Plata. Argentina. . (Organizador) (2005). Trabajo Social latinoamericano 2 A 40 años de la Reconceptualización. Espacio Editorial. Buenos Aires.

AUTORES VARIOS (1983). Trabajo Social en América Latina. Balance y perspectivas. CELATS. Lima.

BADIOU, Alain. (2004). Reportaje en Revista No 56 Diario Clarín. Buenos Aires. 23/10/2004.

CASTEL, Robert. (1997). Las metamorforsis de la cuestión social. Buenos Aires. Paidós.

FALEIROS, Vicente. (1987). "Confrontaciones teóricas de la Reconceptuación". En: Revista Acción Crítica No 21. CELATS. Lima.

. (2003). Estrategias de Empowerment en Trabajo Social. Editorial Lumen-Hvmanitas. Buenos Aires.

FONSECA, Lady. (1982): Una reflexión metodológica" En Revista Acción Crítica Na 12 Lima. ALAETS/CELATS

GRAMSCI, Antonio. (1975). Los intelectuales y la organización de la cultura. Juan Pablos Editor. México D.F.

GRASSI, Estela. (1994). La implicancia de la investigación social en la práctica profesional del Trabajo Social. En: Revista de Treball Social N 135 .. Col-legi Oficial de Diplomats en Treball Social de Catalunya. Barcelona

. (2003). Políticas y problemas sociales en la sociedad neoliberal. La otra década infame (I). Espacio Editorial. Buenos Aires.

IAMAMOTO, Marilda y Raúl DE CARVALHO. (1984). Relaciones Sociales y Trabajo Social: Esbozo de una interpretación histórico-metodológica. CELATS. Lima.

IAMAMOTO, Marilda. (2003). El servicio social en la contemporaneidad. Editora Cortez. Sao Paulo. 
IAMAMOTO, Marilda (2004) As dimensões ético-políticas e teórico-metodológicas no Serviço Social contemporâneo". Trajetória e desafios. En: MOLINA, Ma Lorena (Organizadora.) La cuestión social y la formación profesional en Trabajo Social, en el contexto de las nuevas relaciones de poder y la diversidad latinoamericana. Espacio Editorial. Buenos Aires.

KISNERMAN, Natalio. (1972). Servicio social pueblo. Editorial Hvmanitas. Argentina.

KRUSE, Herman. (1971). "La Reconceptualización del Servicio Social en América Latina". En: Revista Selecciones de Servicio Social No. 13. Editorial Humanitas. Buenos Aires.

LIMA, Boris. (1975). Epistemología del Trabajo Social. Editorial Hvmanitas. Argentina.

LIMA, Leila y RODRÍGUEZ, Roberto. (1977). Metodologismo estallido de una época. No.3 En Revista Acción Crítica. LAETS/CELATS. Lima

LUKÁCS, Gyorgy. (2004). Ontología del ser social: El Trabajo. Ediciones Herramienta. Buenos Aires.

MAGUIÑNA, Alejandrino. (1977).Trabajo Social: iservicio o actividad productiva?. En Revista Acción Crítica. No.3.. ALAETS/CELATS. Lima

MOLINA, Ma Lorena y ROMERO, Ma Cristina. (2001). Los modelos de atención: asistencial, socioeducativo promocional y terapéutico en Trabajo Social. Editorial Universidad de Costa Rica. Costa Rica.

MOLINA, Ma. Lorena (2004). Gestión local desde las municipalidades y desafíos para la formación académica en Trabajo Social, en Costa Rica. En: BURGOS, Nilsa (editora) Gestión local y participación ciudadana.. Espacio Editorial. Buenos Aires

MOLINA, Ma. Lorena. (2004). Derechos humanos y escenarios de la intervención desde el Trabajo Social. En: Revista Costarricense de Trabajo Social. No.16. Costa Rica

MOLINA, Ma Lorena y RUIZ, Ana Isabel. (Now). Recuperando la perspectiva crítico histórica congruente con los fundamentos curriculares. Escuela de Trabajo Social. Universidad de Costa Rica. En RUIZ, Ana Isabel. (Organizadora). Búsquedas Del Trabajo Social Latinoamericano. Espacio Editorial. Argentina. 
MOLINA, Ma. Lorena. (2005). Costa Rica: los caminos recorridos en la formación académica y el trabajo profesional a 40 años de la reconceptualización. En: ALAYÓN, Norberto (Organizador). Trabajo Social latinoamericano - A 40 años de la Reconceptualización. Espacio Editorial. Buenos Aires.

MONTAÑO, Carlos. (1998). La Naturaleza del Servicio Social. Cortez Editora. Sao Paulo.

NETTO, José Paulo. (1981). La crítica conservadora a la Reconceptualización. En: Revista Acción Crítica N 9. CELATS. Lima

NETTO, José Paulo y otros. (1992) La investigación en Trabajo Social. ALAETS /CELATS. Lima.

NETTO, José Paulo. (1992) Capitalismo Monopolista y Servicio Social. Editora Cortez. São Pablo.

PALMA, Diego (1977). La Reconceptualización. Una búsqueda en América Latina. ECRO. Buenos Aires.

PEREIRA, Potyara. (2003). Cuestión social, Servicio Social y derechos de ciudadanía. En: BORGIANNI, Elisabete et al. Servicio Social crítico.. Editora Cortez. São Paulo

QUIIRÓZ, Teresa. (1975). Análisis crítico de los métodos caso, grupo y comunidad.. Instituto de Investigaciones Sociales. Universidad de Costa Rica. Costa Rica

\section{BIOGRAFIA}

\section{NORBERTO ALAYON}

Profesor Licenciado en Trabajo Social, Regular Titular de la Carrera de Trabajo Social de la Universidad de Buenos Aires (UBA). Autor de dieciséis libros y capítulos de libros y de numerosos artículos sobre Trabajo Social y Derechos de la Infancia. Director de la Colección "Desarrollo Social y Sociedad" de la Editorial Espacio de Buenos Aires.

E-mail: nalayon@mail.fsoc.uba.ar

\section{MARIA LORENA MOLINA}

Licenciada en Trabajo Social. Magister Scientiae Administración Pública (Universidd de Costa Roca), Directora de la Escuela de Trabajo Social de la Universidad de Costa Rica. Autora numerosos artículos y libros sobre Trabajo Social y Gerencia Social.

E-mail:mmolina@cariari.ucr.ac.cr 